\title{
Tumor transicional infiltrante de urotelio radioinducido
}

\author{
M. Maroñas Martín, J. Rubio Briones*, L. Arribas Alpuente, P.P. Escolar Perez, \\ M.A. Estornell Gualde, E. Solsona Narbón* \\ Servicios de Oncología Radioterápica y *Urología. Fundación Instituto Valenciano de Oncología. Valencia. \\ Actas Urol Esp 2005; 29 (6): 562-566
}

\section{RESUMEN}

TUMOR TRANSICIONAL INFILTRANTE DE UROTELIO RADIOINDUCIDO

Introducción; El incremento del riesgo de desarrollar un tumor de células transicionales (TCT) en los pacientes tratados con radioterapia y quimioterapia por otras patologías es un hecho conocido, pero muchas veces no tenido en cuenta por su rareza. Nuestro objetivo es revisar el comportamiento clínico de esta asociación en nuestro centro.

Material y métodos; Nuestra casuística de TCT infiltrante con antecedentes de irradiación abdómino pélvica desde 1975. Análisis descriptivo de los casos encontrados con dichos antecedentes.

Resultados; Encontramos 5 pacientes que desarrollaron TCT infiltrante a una media de 19,2 años tras radioterapia (tres de ellos con un seguimiento superior a 20 años y los dos restantes inferior a 10 años). Tres pacientes desarrollaron otro tipo de tumores o lesiones radioinducidas. En dos de ellos coexisten un TCT infiltrante de tracto urinario superior que requirió nefroureterectomía. Los 5 casos desarrollaron TCT de vejiga de alto grado y un paciente desarrolló metástasis por TCT.

Conclusiones; Los pacientes con irradiación abdómino pélvica con un seguimiento prolongado constituyen un grupo de riesgo para el desarrollo de TCT infiltrante. Por tanto, la presencia de hematuria debe alertarnos y exige completar los algoritmos validados para el diagnóstico de TCT en todo el urotelio.

Palabras clave: Carcinoma. Transicional. Radioterapia. Radioinducción.

\section{ABSTRACT}

\section{RADIATION-INDUCED INFILTRATING TRANSITIONAL CELL CARCINOMA}

Introduction: The increased risk of developing a transitional cell carcinoma (TCC) among patients irradiated for other pathologies in a known fact, but many times forgotten due to its low incidence. Our aim is to review the association between radiotherapy (RT) and muscle-infiltrating TCC among our patients.

Material and methods: Clinical survey among our muscle-infiltrating TCC data base since 1975. Descriptive analysis of found cases.

Results: We found 5 patients who developed muscle-infiltrating TCC with a mean time of 19.2 years since radiotherapy (three of them more than 20 years and the other two less than 10 years). Three patients also developed other tumours or pathologies related to radiotherapy. Two of them had an upper tract muscle- infiltrating TCC and required nephroureterectomy. All of them had high risk TCC of the bladder and one developed distant metastasis.

Conclusions: Patients under abdomino-pelvic RT and a prolongued follow-up, can be considered a risk group for developing muscle-infiltrating TCC. Thus, either micro or macrohaematuria or irritative symptoms should lead us to think in this possibility, demanding complete and exhaustive study to rule out TCC in all the urothelium.

Keywords: Carcinoma. Transitional. Radiotherapy. Radioinduction.

$\mathrm{E}$ riesgo de segundas neoplasias tras tratamientos con irradiación y quimioterapia sigue siendo un tema controvertido, ya que además del tratamiento recibido, el riesgo de un segundo tumor puede estar asociado a un estilo de vida o una predisposición genética que puede tener más peso que el antecedente de la irradiación previa ${ }^{1}$. Dentro de la etiología del tumor de células transicionales (TCT) de urotelio son conocidos varios factores carcinogénicos externos como el tabaco o las aminas nitrogenadas. 
Los pacientes tratados con irradiación pueden presentar sarcomas en áreas irradiadas con dosis bajas $^{2}$ como tumores radioinducidos. Es un hecho curioso que entre los supervivientes de ataques nucleares como Hiroshima y Nagasaki, se ven más frecuentemente leucemias y no sarcomas.

Tratamientos de quimioterapia también se han relacionado directamente con tumores de distinta histología. Cabe destacar el Linfoma tipo Hodgkin en el que se han observado mayor riesgo de segundos tumores principalmente de vejiga y leucemias ${ }^{3}$.

Nuestro objetivo es hacer una revisión de los casos acaecidos en nuestro centro de TCT infiltrante en los que se pueda intuir su radioinducción, indagar en su comportamiento clínico y realizar una revisión de la literatura de un hecho clínico que cada vez puede estar más presente en nuestra práctica diaria por el aumento de la población irradiada, la mayor supervivencia de estos pacientes y la coexistencia de otros factores carcinogenéticos del TCT.

\section{CASOS CLINICOS}

Caso 1: Varón de 80 años, no fumador, que fue orquiectomizado a los 52 por seminoma anaplásico, recibiendo RT profunda adyuvante con un total de 5000 rads en campo directo sobre escroto izquierdo y 5.500 rads en el campo retroperitoneal correspondiente. Libre de enfermedad hasta la actualidad, pero a raíz de un cuadro de hematuria a los 28 años de seguimiento fue diagnosticado y resecado un TCT de vejiga GIIT2a (microinvasión con base negativa) con CIS asociado, tratándose con BCG adyuvante manteniéndose libre hasta la actualidad, ocho meses después.

Caso 2: Varón de 52 años, no fumador, orquiectomizado a los 22 por seminoma testicular recibiendo RT profunda adyuvante (se desconocen dosis por provenir de otro Hospital). Libre de enfermedad desde entonces. A los 20 años de seguimiento requirió exéresis y cirugía reconstructiva por radiodermitis y radionecrosis severa en la espalda. A los 24 años de seguimiento, en el estudio por hematuria monosintomática se diagnosticó urográficamente de un tumor de grupo caliciliar superior izquierdo, procediendo a la nefroureterectomía por un TCT de vías GIIpT3.
A los 2 años de la misma presentó recurrencia múltiple a nivel vesical por TCT GIIIT1, recibiendo BCG adyuvante manteniéndose libre de enfermedad hasta la actualidad. A los 29 años de la radioterapia, el paciente fue diagnosticado tras cuadro de ictericia y mediante PAAF de un adenocarcinoma biliopancreático que se ha tratado paliativamente con drenaje biliar, poliquimioterapia y RT paliativa por anemias continuas y profusas secundarias a infiltración tumoral del duodeno. Desde entonces en seguimiento por medicina interna, manteniéndose estable hasta la fecha del estudio.

Caso 3: Varón de 54 fumador que a los 22 sufrió resección intestinal por linfosarcoma intestinal, recibiendo RT adyuvante (se desconocen dosis por provenir de otro Hospital). A los 27 años de seguimiento se le practicaron exéresis de dos carcinomas basocelulares en zona lumbar y gastrectomía total por adenocarcinoma gástrico. Al año siguiente se objetivó anulación funcional del riñón derecho en el contexto de hematurias totales intermitentes, síndrome constitucional y anemia crónica microcítica. Pese a no objetivarse en distintas pruebas radiológicas ningún defecto de repleción y la realización de ureteroscopia descrita como posibles lesiones radicas del uréter, se decide nefroureterectomía derecha por clínica, observándose en la anatomía patológica de la pieza un TCT plano de uréter GIIpT2. Al año de la misma, presenta recurrencia a nivel vesical, resecándose un TCT GIII T1, recibiendo BCG adyuvante, pese a lo cual presentó nueva recurrencia GI Ta en 3 ocasiones durante el seguimiento y actualmente está siendo tratado por metástasis de TCT en hombro derecho mediante RTE antiálgica sobre la zona afecta.

Caso 4: Mujer de 61 años, no fumadora, que a los 52 fue diagnosticada por un carcinoma epidermoide de cérvix moderadamente diferenciado estadio IIa. Se administró Braquiterapia preoperatoria con intervención a las 48 horas (Wertheim - Meigs), sin poder completar la linfadenectomía radical por dificultades técnicas, decidiéndose completar el tratamiento con RTE adyuvante, usando fotones de $10 \mathrm{Mv}$ en dos campos, AP y PA, sobre pelvis y cadenas ilíacas a ritmo de 180 cGy por fracción, hasta una dosis total de 50 Gy. Libre de enfermedad desde entonces. A los 9 años 
de la RT Externa, tras cuadro de hematuria monosintomática, se diagnosticó y resecó un TCT de vejiga, que la AP informó de carcinoma urotelial infiltrante G2 T1.A fecha actual libre de enfermedad.

Caso 5: Varón de 71 años de edad fumador diagnosticado en Enero de 1996 de Adenocarcinoma de próstata, Gleason 5. Se inicia tratamiento neoadyuvante con bloqueo androgénico completo (BAC) durante 3 meses practicándose prostatectomía radical, pT3aNOMO (Gleason $2+4$.). Debido a progresión bioquímica, en Noviembre de 1997 se le practicó biopsia de la anastomosis, con resultado de infiltración por adenocarcinoma, recibiendo RT de rescate en pelvis mediante cuatro campos, hasta una dosis total de 50 Gy con sobreimpresión del lecho quirúrgico de 16 Gy, con fotones de $10 \mathrm{MV}$. El PSA baja a cifras de $0,06 \mathrm{ng} / \mathrm{ml}$. A los dos años del tratamiento RT comienza de nuevo a ascender llegando a cifras de $50 \mathrm{ng} / \mathrm{ml}$, detectándose diseminación ósea a D1, por lo que recibe nuevamente BAC. En Noviembre de 2004 se aprecia ureterohidronefrosis derecha y se practica resección transuretral de un TCT GII infiltrante. Se inicia tratamiento con Quimioterapia, esquema Cisplatino y Gemcitabina. En el momento de la última revisión está pendiente de valorar nueva cistoscopia y rescate quirúrgico.

\section{DISCUSION}

Hay que reseñar la dificultad que compete el estudio de la radioinducción de los TCT por diversos motivos. En primer lugar, la mayoría de las publicaciones son observacionales y no son planteables ningún otro tipo de estudios por la necesidad de un largo seguimiento de los pacientes irradiados. Además la concomitancia de otros factores carcinogenéticos (dos de nuestros pacientes eran fumadores), la posible coexistencia de mutaciones génicas preexistentes y la ausencia en la mayoría de los casos de un estudio genotípico completo no permiten aseverar la radioinducción como factor carcinogenético único a ciencia cierta.

El concepto de oncogénesis por radiación fue establecido por Cahan y colaboradores en 1948, cuando describió 11 casos de sarcomas secun- darios óseos inducidos tras radioterapia por tumores óseos y de mama, estableciendo unos criterios para considerar una neoplasia como radioinducida 1) aparición de la segunda neoplasia dentro del área irradiada, 2) debe existir un periodo de latencia entre la irradiación y el diagnostico tumoral (habitualmente años), 3) la nueva neoplasia debe ser histológicamente distinta, 4) el paciente no debe tener predisposición genética para el desarrollo tumoral ${ }^{5-7}$.

Hay bastante evidencia que los cánceres en los humanos, pueden ser el resultado bien de la activación de un oncogen dominante, o de la pérdida de un gen supresor. Se conoce que los oncogenes pueden ser activados por una mutación puntual, una traslocación cromosómica o por la amplificación de un gen. La radiación produce supresiones y traslocaciones cromosómicas más que mutaciones puntuales. Estos mecanismos son posibilidades atractivas para explicar el mecanismo por el cual la radiación puede provocar un cáncer, pero no han sido demostrados en ningún tumor humano "radioinducido". La transformación de un gen dominante ha sido observada en una transformación oncogénica in vitro, pero el gen no se ha podido todavía secuenciar. La activación del K-RAS N- RAS ha sido publicada en una proporción de linfoma murino tímico inducido por rayos $\mathrm{X}$ o neutrones ${ }^{3}$.

Se ha demostrado in vitro el poder carcinogenético de la radioterapia sobre cultivos celulares de urotelio sano o líneas celulares transicionales. Así quedó demostrado en el estudio de Antonakopoulos en 1982, quien estudió el efecto de la radiación sobre la pared vesical en ratas con una dosis de 20 Gy. A los seis meses postirradiación se veían focos de hiperplasia, inicialmente sólo en las células básales, extendiéndose después sobre la capa intermedia. A los 20 meses postirradiación se vieron TCT en 10 de los 17 animales supervivientes (59\%); Además de un intensa fibrosis que afectaba no sólo al epitelio transicional de la pared vesical, sino también a la capa muscular de la misma.

Sin embargo, la ratio de TCT de vejiga observada/esperada en pacientes que han recibido radioterapia en el tratamiento de los tumores germinales de testículo ha sido 0,98, 1.80, 2,04, 2,07 y 2,76 a los 4, 9, 14, 19 y más de 20 años 
de seguimiento respectivamente, con 369 TCT de vejiga observados entre 3306 pacientes seguidos más de 20 años por este tipo de tumores ${ }^{9}$. Aún no se ha evaluado si el cisplatino, el agente quimioterápico que centra el tratamiento del tumor testicular diseminado y a su vez conocido como potenciador de la radioterapia, pudiera adelantar la aparición de TCT inducidos por ésta. También en las pacientes irradiadas por tumores ginecológicos (cérvix, endometrio) el riesgo de TCT es mayor que en población normal. Boice y colaboradores, en el año 1985, estudiaron el riesgo de segundos tumores en pacientes que habian recibido RT sobre campo pélvico en el contexto de un carcinoma de cérvix, llegando a la conclusión que estas pacientes tenían un mayor riesgo de desarrollar cáncer de vejiga (con $R R$ de 4,5 ), recto $(R R=1,8)$ y vagina $(R R=2,7)$ entre otros. (Boice ${ }^{\prime} 88$ y Travis`96 en Travis'99).

Cuando se pueden estudiar un suficiente número de pacientes tras recibir tratamientos de radioterapia, con los controles adecuados, la irradiación parece inducir una pequeña pero significativa incidencia de segundos tumores en los tejidos que han recibido toda la dosis, así como en órganos alejados del volumen "blanco" irradiado que han recibido pocos Gy. La radiación es un conocido agente carcinogénico, y no nos debemos sorprender de la aparición de segundos tumores, que en general está relacionada con el 5 $\%$ de los segundos tumores que aparecen en el seguimiento ${ }^{3}$.

Tras tratamientos con radioterapia por tumores de próstata, Brenner et al. describen un mayor riesgo de segundos tumores de cualquier tipo y localización, significativamente mayor tras irradiación que tras cirugía, sobre un $6 \%$, incrementándose el riesgo relativo llegando a ser del $34 \%$ a 10 años ${ }^{2,12}$.

En nuestra serie de 237 tumores de próstata tratados con RT de 1.990 a 1.999, 96 de ellos con RT como monoterapia exclusiva, la tasa de segundos tumores es del 17,7 \%; si la modalidad terapéutica es de RT y Hormonoterapia concomitante (114 pacientes), el porcentaje es el 12,3\%; si la RT es utilizada como rescate tras cirugía ( 27 pacientes), los segundos tumores representan el $7,4 \%$. Dentro del global de pacientes irradiados por cáncer de próstata, existen 14 pacientes con
TCT superficial que no han sido incluidos en este trabajo; 7 de ellos por presentarlo previamente a la RT-justificando así la interrelación de la predisposición genética o de los antecedentes personales expuesta previamente-, y 7 porque han sido TCT superficiales con menos de 3 años de seguimiento tras la RT, lo que pensamos que es un hecho diferencial claro frente al grupo de pacientes motivo de este estudio (infiltrantes y larga evolución) y que hace poner más en duda la radioinducción de los mismos.

De la misma forma que en el seguimiento de los pacientes irradiados que presentan rectorragias hacemos colonoscopia aunque la rectitis sea la causa más frecuente (además de controlar el CEA), ante micro o macrohematuria, clínica miccional o dolor lumboabdominal, ponemos en marcha los algoritmos pertinentes para descartar causa tumoral (ecografía, citología, urografía y endoscopia incluyendo si es necesario el tracto urinario superior).

Con la incorporación de las nuevas técnicas de la Radioterapia moderna como la IMRT (RT con intensidad modulada), con la cual podemos administrar dosis de radiación más altas a un volumen más reducido alrededor del tumor, alcanzando dosis menores en los órganos críticos vecinos, reducimos considerablemente los efectos agudos. Cabría pensar que los efectos carcinogenéticos también deberían ser menores, no obstante Hall y colaboradores han publicado recientemente un artículo en el que defienden la hipótesis contraria ya que con la IMRT se aumenta el volumen de tejido normal expuesto a dosis menores de irradiación, produciendo un incremento en un 0,5\% del riesgo de tumoración radioinducida. Asimismo se requieren mayores unidades monitor para conseguir la distribución preestablecida, siendo la dosis por fugas y radiación dispersa mayor, dicho factor incrementa un 0,25\% adicional al riesgo anterior, por lo que la posibilidad del tumor radioinucido por la IMRT es de un $0,75 \%{ }^{13,14}$. Followill y colaboradores han publicado que el riesgo estimado de la IMRT frente a la RT convencional oscila entre un 0,4 y un $1 \%$ de incremento añadido, sobre todo si utilizan fotones de $18 \mathrm{MV}$ - aunque con la IMRT actual, la energía recomendada es de 6 MV-5. 
La importancia del tiempo de seguimiento es clara; Smith y Doll (1968-76), en un seguimiento a 10 años de pacientes irradiadas sobre campo pélvico, no mostraron relación alguna entre TCT y Radioterapia ${ }^{6}$. Brinkley y Habbitle en 1969, en un seguimiento a 15 años tampoco logró demostrar relación ninguna. Fue en 1977, cuando Duncan realizó un seguimiento de 25 años en el que sí logró demostrar esta relación entre radiación pélvica y TCT. Concluyendo, por tanto, que se requieren periodos mínimos de 20-25 años para demostrar esta relación.

En el estudio de Quilty en 1987, se recogieron once mujeres, seguidas desde 1971, con cáncer vesical postirradiación pélvica. En la mayoría de ellas el cáncer era de comportamiento altamente agresivo, similar a nuestra casuística, haciendo un pronóstico realmente pobre con sólo un $32 \%$ de supervivencia al año. Se observó también clara diferencia en el tiempo de evolución desde la irradiación pélvica a la aparición del tumor vesical en relación con la dosis de radiación recibida, siendo esta diferencia de 30 años en el caso de baja dosis de radiación y de 16,5 años en el caso de alta dosis de radiación ${ }^{1}$.

Entre nuestros pacientes cabe destacar que la aparición de TCT ha sido a una media de 19,2 años de seguimiento desde la RT, que en 2 casos han sido TCT de alto grado e infiltrantes de tracto urinario superior y que los 5 casos han desarrollado TCT de vejiga de alto riesgo, lo que supone que la aparición de TCT en estos pacientes conlleva un riesgo de progresión a distancia alto, como el demostrado en el caso 3.

Nos parece obligado por todo ello el seguimiento urológico de todos los pacientes irradiados por tumores abdominales o pélvicos de otra extirpe. La presencia de sintomatología urológica o hematuria macro o microscópica nunca debe ser justificada exclusivamente por posibles lesiones rádicas benignas, debiendo descartar una segunda neoplasia, por lo que nuestros algoritmos de seguimiento deben incluir el estudio con imagen o endoscópico, para descartar o confirmar un TCT en todo el tracto urinario, pues en muchos casos, como el caso 3 , son tumores no exofíticos difíciles de diagnosticar.

\section{CONCLUSIONES}

Los pacientes irradiados por tumores abdominales o pélvicos y con un seguimiento largo tienen un riesgo mayor de desarrollar tumor de células transicionales infiltrantes. El seguimiento urológico y digestivo de estos pacientes debe se regular, a largo plazo y exhaustivo ante cualquier signo o síntoma urológicos.

\section{REFERENCIAS}

1. Brenner DJ, Curtis RE, Hall EJ, Ron E. Second malignancies in prostata carcinoma patients after radiotherapy compared with surgery. Cancer 2000;88:398-406.

2. Boice JD, Day NE, Anderson A, et al. Second cancers following radiation treatment for cervical cancer: An international collaboration among cancer registries. J Natl Cancer Inst 1985; 74:955-975.

3. Cahan WG, Woodard HQ, Higonbotham NL, et al. Sarcoma arising in irradiated bone. Report of eleven cases. Cancer 1948; 1:3-29.

4. Antonakopoulos GN, Hicks RM, Hamilton E, Berry RJ. Early and late morphological changes (including carcinoma urothelium) induced by irradiation of the rat urinary bladder. $\mathrm{Br} \mathrm{J}$ Cancer 1982;46:403-416.

5. Lois B. Travis, Rochelle E, Curtis, et al. Risk of second malignant neoplasms among long-term survivors of testicular cancer. Journal of the National Cancer Institute 1997; 89:14291439.

6. Hall E, Cheng-Shie W. Radiation-Induced second cancers: The impact of 3D-CRT and IMRT. Int $\mathrm{J}$ Radiat Oncol Biol Phys 2003;56:83-88.

7. Hall E. Principles of Carcinogenesis: Physical. In De VitaVT, Hellman S, Rosenberg SA, Cancer Principles \& Practice of Oncology Vol. 1. JB Lippicont Company Philadelphia 1993: 213-227.

8. Followill D, Geis P, Boyer A. Estimates of Whole-Body dose equivalent produced by beam intensity modulated conformal therapy. Int J Radiat Oncol Biol Phys 1997;38:667-672.

9. Smith PG, Doll R. Late effects of $x$ irradiation in patients treated for metropathia haemorrhagica. Br J Radiol 1976;49: 224-232.

10. Duncan RE, Bennett DW, Evans AT. Aron BS, Schellhas HF. Radiation-induced bladder tumors. J Urol 1977;118: 43-45.

11. Quilty PM, Kerr GR. Bladder cancer following low or high dose pelvic irradiation. Clin Radiol 1987;38:583-585.

12. Takanobu K, Tohru H, Rynosuke U, et al. J Neurosurg 2.001; 95: 710- 713 .

13. McIver J, Pollock BE. Radiation-induced tumor after stereotactic radiosurgery and whole brain radiotherapy: case report and literature review. Journal of Neuro-Oncology 2.004: 66:301-305.

14. Kedar N. Prasad. Radiation Carcinogenesis: Animal model. In CRC Handbook of Radiobiology. CRC Press, Boca Raton Florida 1984 : 213-226.

Dr. J. Rubio Briones

Servicio de Urología. IVO.

C/ Prof. Beltrán Báguena 8 - 46009.Valencia

e-mail: jrubio@fivo.org

(Trabajo recibido el 14 febrero de 2005) 